\title{
Efficient silicon based light emitters
}

\author{
M. Helm*, J.M. Sun, J. Potfajova, T. Dekorsy, B. Schmidt, W. Skorupa \\ Institute of Ion Beam Physics and Materials Research, Forschungszentrum Rossendorf, P.O. Box 510119, 01314 Dresden, Germany
}

\begin{abstract}
Recent progress on electrically driven silicon based light emitters is reviewed, with emphasis on our work on light emitting pn diodes (LED) and MOS devices doped with rare-earth elements. The LEDs were fabricated by high-dose boron implantation, producing nanoscale modifications in the material. The electroluminescence (EL) efficiency increases with temperature, reaching $0.1 \%$ (wall plug efficiency) at room temperature for optimized conditions. Such devices were integrated into a microcavity. In the MOS devices, the oxide was implanted with various rare-earth elements, resulting in strong EL in the visible ( $\mathrm{Tb}$ ) and ultraviolet $(\mathrm{Gd})$. External quantum efficiencies in excess of $10 \%$ are reported.
\end{abstract}

Keywords: Silicon light emitters; Ion implantation; Rare earth; Microcavity; Electroluminescence

\section{Introduction}

Many optoelectronic components, such as waveguides, modulators [1] and detectors, can be fabricated from silicon or related materials $\left(\mathrm{SiO}_{2}, \mathrm{SiGe}\right)$. The only component missing for monolithically integrated Si optoelectronics [2] is an efficient light emitter, be it an LED or a laser. Its most likely use would be in optical interconnects between and within microelectronic chips. This so called 'interconnect bottleneck' may become severe in the near future due to excessive signal delays and overheating, which are a result of the higher transistor density in every new micro(and nano-) electronic generation. Note that an optically pumped Si Raman laser has been demonstrated recently [3] — certainly a big step forward, but what is ultimately needed is an electrically pumped source.

In common experience, $\mathrm{Si}$ is an inefficient light emitter due to its indirect bandgap. In pure $\mathrm{Si}$, the radiative relaxation time is longer than milliseconds, much slower than recombination via defects states (Shockley-Read-Hall recombination) or Auger recombination at higher injection or doping, which results in efficiencies of the order of $10^{-6}$. Every approach to make a more efficient light emitter must either increase the radiative recombination rate or suppress

\footnotetext{
* Corresponding author. Tel.: + 49351 2602260; fax: +493512603285. E-mail address: m.helm@fz-rossendorf.de (M. Helm).
}

the non-radiative channels. The former approach is of course the preferable one, since it would also offer a faster device response time.

Starting around 1990 with the discovery of red luminescence from porous Si [4], several approaches have been developed to enhance Si luminescence, many of which are related to nanoscale material modifications. While for some applications porous $\mathrm{Si}$ is well suited (e.g. Bragg mirrors can be easily fabricated by periodically varying the etch rate $[5,6])$, it suffers from insufficient long-term stability and is not easily compatible with standard microelectronic processing technology [7]. Quantum confinement, and thus a relaxing of the k-selection rules, can also be achieved in $\mathrm{Si} / \mathrm{SiGe}$ quantum wells. Although improved photo- and electroluminescence was observed [8], the efficiency was quenched significantly for higher temperatures up to $300 \mathrm{~K}$.

A very promising approach is based on Si-rich $\mathrm{SiO}_{2}$, or $\mathrm{Si}$ nanocrystals in an $\mathrm{SiO}_{2}$ matrix, realized in the oxide of an MOS structure either by ion implantation or PECVD growth of the oxide [9]. In these structures, the luminescence wavelength is an indicator of the nanocrystal size. Very recently, optical gain has even been observed under strong optical pumping [10]. It is suspected that the observed gain results from states at the interface between the nanocrystals and the $\mathrm{SiO}_{2}$ matrix. Yet the challenge is now to transfer this mechanism to electrical excitation.

Other promising approaches make use of embedding the rare-earth element erbium into a matrix and employing 
the famous $\mathrm{Er}^{3+} 4 \mathrm{f}$-intrashell transition at $1.54 \mu \mathrm{m}$ [11], which, e.g. is used in optically pumped fiber amplifiers. Electrical excitation, however, requires a conducting host material. Therefore it has been attempted to introduce $\mathrm{Er}^{3+}$ ions into $\mathrm{Si}$. In this way, light emitting diodes (LEDs) were demonstrated [12], but the efficiency also dropped at higher temperature $(300 \mathrm{~K})$ due to a back transfer process. Yet it is also possible to introduce rare-earth ions into the oxide of a Si MOS structure, which can then be excited by applying a voltage between the gate and the substrate. Below we will describe strong electroluminescence from MOS devices implanted with Gd and Tb, emitting UV light at $316 \mathrm{~nm}$ [13] and green light at $541 \mathrm{~nm}$ [14], respectively. This opens the way for applications of Si-based light emitters for the analysis of biological substances or low-cost microdisplays.

The luminescence from rare-earth ions has been combined with the nanocrystal concept. It turns out that the Si nanocrystals act as 'sensitizers' for the $\mathrm{Er}^{3+}$, i.e. they help to provide an efficient energy transfer to the $\mathrm{Er}^{3+}$ ions, thus enabling efficient MOS type LEDs emitting at $1.54 \mu \mathrm{m}$ [15-18].

Finally there have been also recent remarkable observations of enhanced electroluminescence (EL) in $\mathrm{Si}$ pn diode structures. In one approach, methods known from solar-cell technology were utilized, such as employing ultraclean float-zone Si substrates, improved surface passivation and applying surface texture to outcouple a larger fraction of the generated photons [19]. Since this method attempts to suppress the non-radiative relaxation channels, the device response will be slow ( $>\mathrm{ms}$ ). In another approach, boron was implanted into n-type $\mathrm{Si}$ at a high dose, and the resulting pn diodes were shown to exhibit increasing EL efficiency with increasing temperature [20]. The efficient EL was assumed to result form carrier confinement near dislocation loops formed after implantation and annealing.

We will describe below our recent progress related to this latter approach. In our B-implanted structures, the electroluminescence (EL) increases with temperature, resulting in a wall-plug efficiency of $0.1 \%$ at room temperature [21]. In addition, we have integrated these LEDs into a microcavity [22].

\section{Boron implanted Si light emitting pn diodes}

The silicon pn diodes were prepared by high-dose boron implantation into (001) oriented Sb-doped n-type silicon substrates with a resistivity of $0.1 \Omega \mathrm{cm}$. Boron doses between $2 \times 10^{13}$ and $3 \times 10^{17} \mathrm{~cm}^{-2}$ were implanted at an energy of $25 \mathrm{keV}$. All samples were subsequently furnace annealed at $1050{ }^{\circ} \mathrm{C}$ for $20 \mathrm{~min}$ and processed into $1 \mathrm{~mm}$ diameter diodes with aluminum ring contacts on top.

Cross-section transmission electron microscopy (XTEM) reveals implantation induced defects (see Ref. [23]) as well as individual dark spots with a diameter of $10-20 \mathrm{~nm}$ at

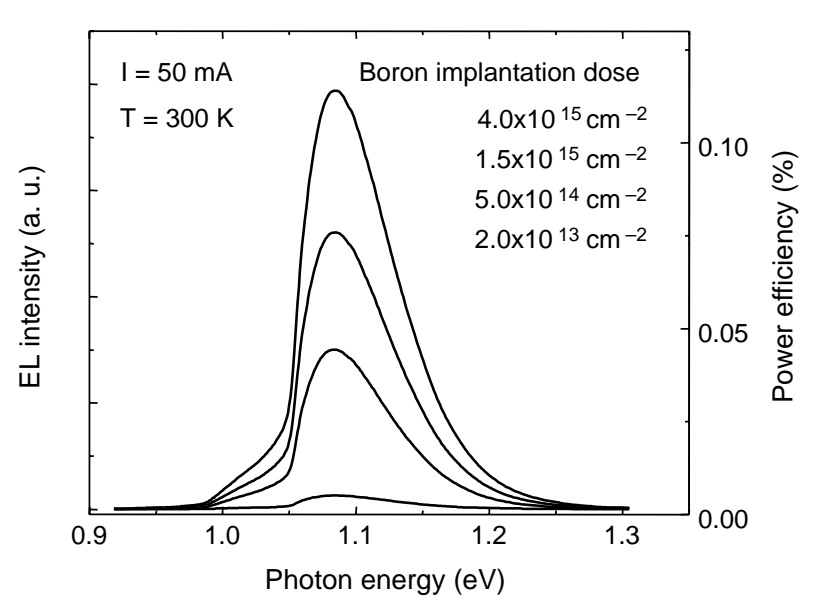

Fig. 1. Room-temperature electroluminescence spectrum for samples with different B implantation doses (lowest/highest curve corresponds to lowest/highest dose, respectively).

the end of curved dislocation lines close to the pn junction after annealing. Their origin is attributed to high strain in an area of locally high density of $\mathrm{Si}-\mathrm{B}$ interstitial clusters, $\mathrm{Si}$ interstitials or to strain fields around extended defects. Energy dispersive X-ray spectroscopy (EDX) using a $70 \mathrm{~nm}$ focused electron beam reveals a high boron concentration at the dark spots compared to the defect free regions. Such a localized high boron concentration is attributed to boron gettering during the nucleation of the extended defects. We believe that these regions act as locally enhanced doping (doping spikes).

Fig. 1 shows the room temperature EL spectra for samples with different implantation doses. Obviously higher boron doses result in stronger EL, reaching a power conversion efficiency of over $0.1 \%$ for a dose of $4 \times$ $10^{15} \mathrm{~cm}^{-2}$ (at even higher doses, the efficiency drops again somewhat, see Ref. [21]).

More physical information can be gained from the lowtemperature EL spectra, shown in Fig. 2 for different implantation doses. The spectra exhibit a peak from

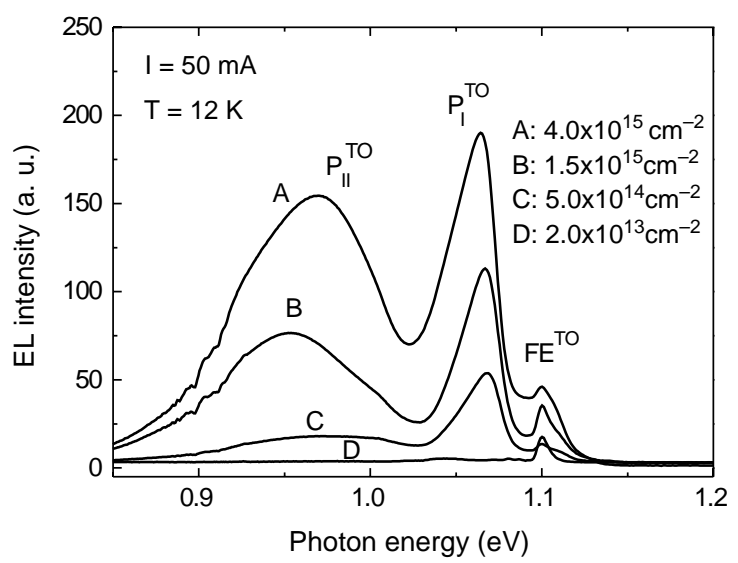

Fig. 2. Low-temperature electroluminescence spectrum for samples with different $\mathrm{B}$ implantation doses. The peaks $\mathrm{P}_{\mathrm{I}}^{\mathrm{TO}}, \mathrm{P}_{\mathrm{II}}^{\mathrm{TO}}$, and $\mathrm{FE}^{\mathrm{TO}}$ are indicated. 
the transverse optical (TO) phonon-assisted free exciton recombination at $1.1 \mathrm{eV}\left(\mathrm{FE}^{\mathrm{TO}}\right)$ and two broader asymmetric EL peaks close to 1.05 and $0.95 \mathrm{eV}$ from TO phononassisted recombination of excitons bound to traps, $\mathrm{P}_{\mathrm{I}}^{\mathrm{TO}}$ and $\mathrm{P}_{\mathrm{II}}^{\mathrm{TO}}$, respectively.

At the lowest implantation doses of $2 \times 10^{13} \mathrm{~cm}^{-2}$, no luminescence from bound excitons is observed in the EL spectrum. Above an implantation dose of $5 \times 10^{14} \mathrm{~cm}^{-2}$, $\mathrm{P}_{\mathrm{I}}^{\mathrm{TO}}$ and especially $\mathrm{P}_{\mathrm{II}}^{\mathrm{TO}}$ increase rapidly with further increasing the boron doses. This is the regime where visible extended defects are observed by XTEM. The maximum EL is reached at a dose of $4 \times 10^{15} \mathrm{~cm}^{-2}$, where the $\mathrm{B}$ concentration is already higher then the B solubility at the annealing temperature. At even higher dose the B clusters cannot be dissolved into doping spikes anymore. These results suggest that both peaks are related to the traps created by high-dose B implantation and the subsequent annealing (for details, see Ref. [23]).

The peak height of the bound-exciton peaks $\mathrm{P}_{I}^{\mathrm{TO}}$ and $\mathrm{P}_{\mathrm{II}}^{\mathrm{TO}}$ as well as the overall integrated EL intensity of the $\mathrm{FE}^{\mathrm{TO}}$ peak and its phonon replicas is plotted as a function of temperature in Fig. 3. The $\mathrm{P}_{\mathrm{I}}^{\mathrm{TO}}$ peak decreases from $15 \mathrm{~K}$ and is completely thermally quenched at $80 \mathrm{~K}$, while the $\mathrm{P}_{\mathrm{II}}^{\mathrm{TO}}$ peak starts to decrease at $80 \mathrm{~K}$ and is thermally quenched at a temperature of $260 \mathrm{~K}$. Associated with the decrease of these two peaks is an increase of the $\mathrm{FE}^{\mathrm{TO}}$ peak. This anomalous increase of the band-edge EL intensity with temperature is in contrast to the temperature dependence of the PL from the n-type substrate, which shows no traprelated luminescence. The EL intensity of the $\mathrm{FE}^{\mathrm{TO}}$ peak shows a two-step increase with rising temperature in close correlation with the decrease of the two bound-exciton peaks. This correlation indicates that the increase of the band-edge free electron-hole recombination comes from the thermal dissociation of bound excitons with increasing

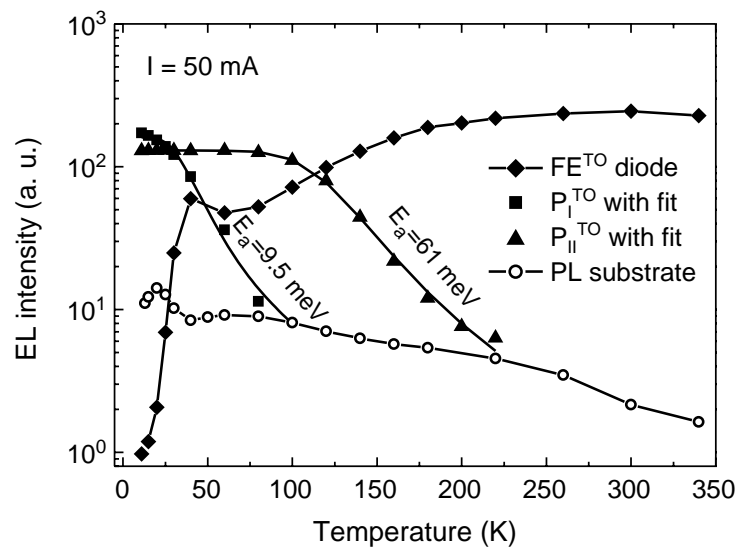

Fig. 3. Temperature dependence of the different electroluminescence peaks. While the $\mathrm{FE}^{\mathrm{TO}}$ line (diamonds with line as guide for the eye) shows a twostep increase with temperature, the $\mathrm{P}_{\mathrm{I}}^{\mathrm{TO}}$ and $\mathrm{P}_{\mathrm{II}}^{\mathrm{TO}}$ lines decrease with an activation energy of 9.5 and $61 \mathrm{meV}$, respectively (lines are theoretical fit). In addition, the temperature dependence of the substrate photoluminescence is shown (open circles), showing a decrease with temperature. temperature. Our results can be well reproduced with a rate equation model [23], taking into account the excitons bound to the two traps and the free excitons/electron-hole pairs. A fit yields activation energies of 9.5 and $61 \mathrm{meV}$, respectively, as shown in Fig. 3. The same model allows us to accurately describe the EL vs current behavior at all temperatures as well as the current-voltage characteristic, which exhibits an S-shaped bistability at low temperature [24].

An important observation (not shown) is a blue-shift of both bound-exciton EL peaks, whose magnitude is proportional to the logarithm of the injection current. Such a behavior is typical for screening of a doping-induced potential (see Ref. [25] for a similar behavior) and it corroborates the assumption that the traps are related to boron doping spikes. We then attribute $\mathrm{P}_{\mathrm{I}}^{\mathrm{TO}}$ and $\mathrm{P}_{\mathrm{II}}^{\mathrm{TO}}$ to unstrained and strained boron-rich areas [23], respectively (strain would reduce the effective band gap, thus explaining the relative spectral positions, see also Ref. [26]). We would like to note that a reference sample, implanted with high doses of $\mathrm{Si}$ instead of $\mathrm{B}$, exhibits an entirely different behavior in all respects described above [23], which excludes bare dislocations as the origin of our observations and supports the assumption of doping spikes.

Although it may not be possible to make a pn-junction laser based on the present concept (or not at all), a significant improvement can be achieved by employing a resonant cavity, which is known to lead to a better outcoupling efficiency and directionality of the emitted radiation. This concept has been also demonstrated for $\mathrm{Si}$ based light emitting devices, but mostly for optical excitation [27,28] (photoluminescence), apart from a porous-Si diode operated with a relatively high driving voltage [6]. We present here an electrically pumped resonant-cavity LED, which is based on the above Si pn diodes.

The device (Fig. 4) consists of a buried $\mathrm{CoSi}_{2}$ layer, which acts as the bottom mirror and the bottom electrical contact, the active layer containing the pn diode, and a top Bragg mirror consisting of 2.5 pairs of $\mathrm{Si} / \mathrm{SiO}_{2}$. The $\mathrm{CoSi}_{2}$ layer is formed by ion beam synthesis based on $\mathrm{Co}^{+}$ implantation into n-doped (100) $\mathrm{Si}$ and subsequent annealing [22]. The top crystalline $\mathrm{Si}$ layer is overgrown using molecular beam epitaxy to a total thickness of $370 \mathrm{~nm}$, corresponding to a $\lambda$-cavity at a wavelength of $1115 \mathrm{~nm}$. The Si layer is uniformly $\mathrm{n}$-doped $\left(1.8 \times 10^{17} \mathrm{~cm}^{-3}\right)$ by implanting $\mathrm{P}^{+}$ions and subsequent annealing. The Si pn diode is formed by $\mathrm{B}^{+}$ion implantation $(25 \mathrm{keV}$ energy, dose of $4 \times 10^{15} \mathrm{~cm}^{-2}$ ) and high temperature annealing $\left(1050{ }^{\circ} \mathrm{C}\right.$ for $\left.10 \mathrm{~min}\right)$ into the MBE grown $\mathrm{n}$-doped Si top layer.

Fig. 5 depicts the room-temperature EL spectrum of devices without and with Bragg mirror at a current of $500 \mathrm{~mA}$. Additionally the reflectivity spectrum measured within the $\mathrm{Al}$ ring contact of a diode is shown, exhibiting the clear signature of a microcavity. The top Bragg mirror has a reflectivity of $94 \%$. At the position matching $\lambda=1115 \mathrm{~nm}$ 


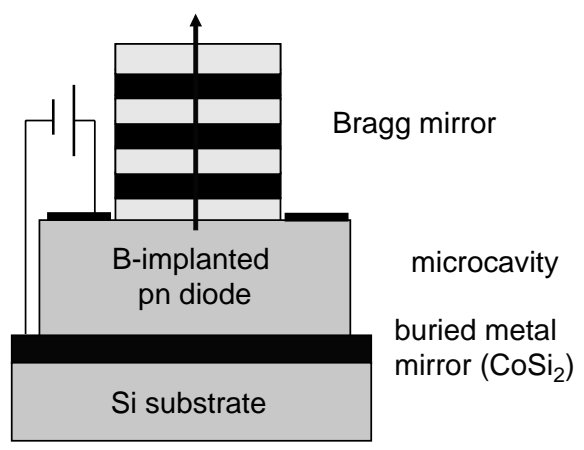

Fig. 4. Schematic of the resonant-cavity LED.

the reflectivity drops to $60 \%$. This spectrum agrees with the modeling of the microcavity using a value of $75 \%$ for the reflectivity of the $\mathrm{Si} / \mathrm{CoSi}_{2}$ interface. The bandgap EL spectrum of the device without Bragg mirror shows essentially the same spectrum as observed for the devices discussed above (cf. Fig. 1) with a linewidth of $84 \mathrm{~nm}$. With the top-Bragg mirror the linewidth is decreased to $35 \mathrm{~nm}$ while the peak intensity is significantly increased at the resonance. The slight shift of the EL maximum with respect to the reflectivity minimum is probably due to a non-uniform thickness of the Bragg mirror across the wafer.

The present device represents a proof-of-principle, however, it has a rather poor electrical characteristic and emission efficiency. This is due to insufficient quality of the MBE grown $\mathrm{Si}$ layer in this non-optimized fabrication process and excessive B diffusion, which is supported by SIMS measurements. Presently we are attempting a fabricating scheme based on wafer bonding, which could result in a significant performance improvement. Another approach could be the integration into a photoniccrystal structure, in order to suppress in-plane light propagation $[29,30]$.

Finally we wish to note that stimulated emission from an edge-emitting Si pn junction has recently been reported [31], yet still awaiting confirmation by other groups.

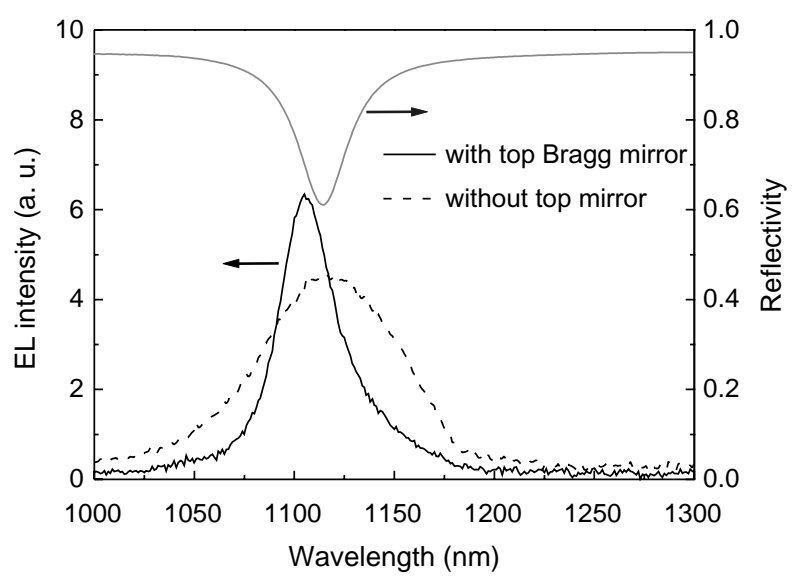

Fig. 5. Electroluminescence spectrum of the Si LED with and without Bragg mirror on top. For comparison, the reflectivity spectrum of the microcavity is also shown.

\section{Rare-earth doped Si-MOS light emitters}

Rare-earth doping is a common technique to fabricate fluorescent devices for display applications. Since the energy gap of the host material has to be larger than the active transition energy, wide-gap semiconductors such as $\mathrm{GaN}$ and $\mathrm{ZnS}$ have been used for dopants emitting visible light. Examples are GaN:Eu,Tm [32] or ZnS:Tb [33]. Also doping of other insulators or glasses has been employed, the most famous example probably being the Er doped fiber amplifier used in optical long-distance communications [11]. While Er is probably the most widely studied dopant, recently also doping of MOS structures with other rare-earth elements $(\mathrm{Tb}, \mathrm{Yb})$ has been demonstrated [17]. Here we report on very bright-green Tb doped MOS light emitters [14] as well as deep-ultraviolet MOS emitters doped with $\mathrm{Gd}$ [13]. $\mathrm{Gd}^{3+}$ has a luminescent transition at a wavelength of $316 \mathrm{~nm}$ and therefore needs a very-large band-gap host material [34]. Presently, deep UV LEDs are mostly based on $\mathrm{AlGaN}$ [35-37], whereas for the green LEDs InGaN [38] or AlGaInP [39] are employed. The substitution of those materials by Si MOS structures would be highly desirable for low-cost and integrated applications.

Devices were prepared by standard Si MOS technology on 4-inch $\{100\}$ oriented n-type silicon wafers with resistivity of $2-5 \Omega \mathrm{cm}$. The MOS structures were fabricated by local oxidation of silicon (LOCOS) with gate oxide and a field oxide thickness of $1 \mu \mathrm{m}$, as shown in Fig. 6. The active layer in the gate oxide is a $100 \mathrm{~nm}$ thick thermally grown $\mathrm{SiO}_{2}$ implanted with $\mathrm{Gd}^{+}$or $\mathrm{Tb}^{+}$ions at different energies and doses to yield a flat concentration profile over $20-30 \mathrm{~nm}$ in the gate oxide. The peak concentration of the rare earth elements was varied between 0.05 and $3 \%$. The implantation was followed by furnace annealing at 800 $1100{ }^{\circ} \mathrm{C}$ in flowing $\mathrm{N}_{2}$ for $1 \mathrm{~h}$. The gate electrode is a $100 \mathrm{~nm}$ transparent indium-tin-oxide layer deposited by RF sputtering. Various shapes of MOS devices with different feature size in the range from 2 to $500 \mu \mathrm{m}$ were fabricated.

EL spectra were measured on a $500 \mu \mathrm{m}$ diameter device at a constant current. The EL signal was recorded at room temperature with a monochromator and a photomultiplier, the absolute EL power from the device was measured using a calibrated optical power meter.

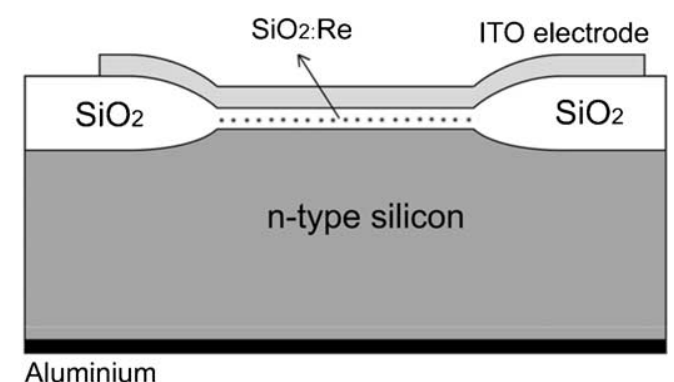

Fig. 6. Schematic of rare-earth doped MOS light emitting device. 


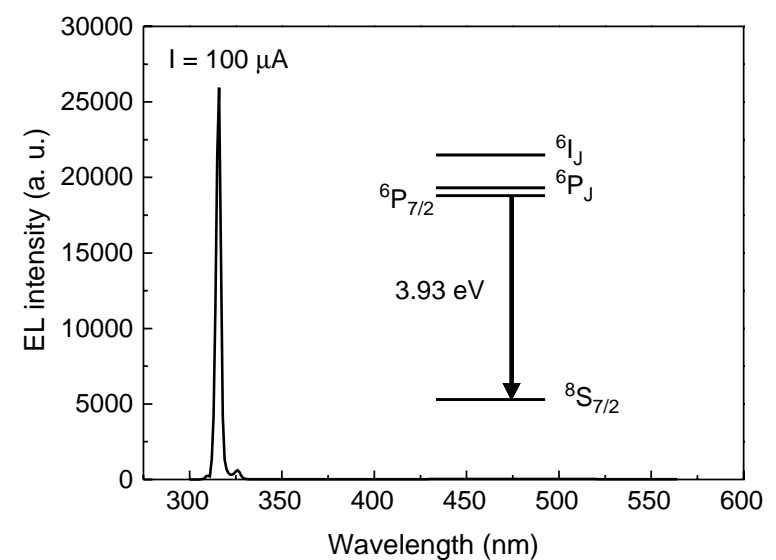

Fig. 7. Electroluminescence spectrum of the Gd doped MOS device at $100 \mu \mathrm{A}$ current and a sketch of the few lowest energy levels in $\mathrm{Gd}^{3+}$.

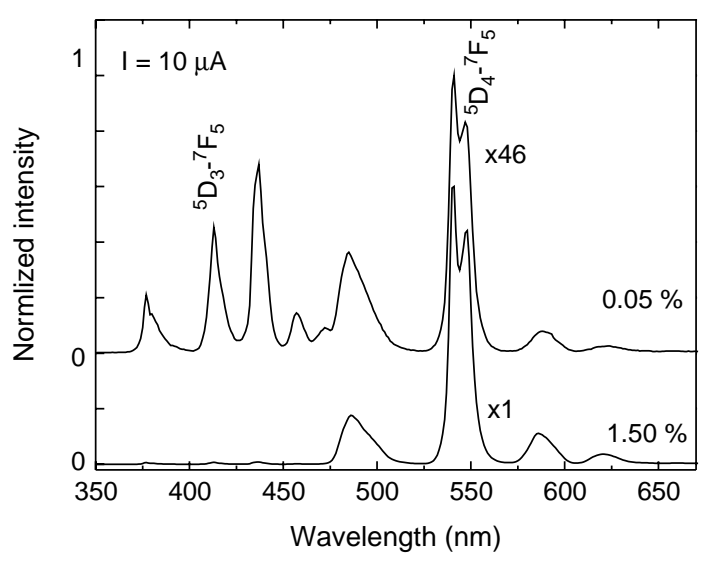

Fig. 8. Electroluminescence spectrum of Tb doped MOS devices with two different $\mathrm{Tb}$ concentrations at a current of $10 \mu \mathrm{A}$. The spectrum at $0.05 \%$ has been multiplied by 46 , to display the same maximum peak height as the $1.5 \%$ sample.

Fig. 7 shows the EL spectra of the $\mathrm{SiO}_{2}: \mathrm{Gd}$ at an injection current of $100 \mu \mathrm{A} . \mathrm{Gd}^{3+}$ has a rather singular position among the trivalent rare-earth ions, since it has a ground state with S-type symmetry, with the lowest excitation in the ultraviolet. Hence the spectra show a strong single peak at $316 \mathrm{~nm}$ from the transition of the first excited state ${ }^{6} \mathrm{P}_{7 / 2}$ to the ${ }^{8} \mathrm{~S}_{7 / 2}$ ground state.

The $\mathrm{Tb}^{3+}$ ion exhibits a series of transitions from the ${ }^{5} \mathrm{D}_{3}$ and the ${ }^{5} \mathrm{D}_{4}$ state, respectively, to the ground state multiplet of ${ }^{7} \mathrm{~F}_{J}$ states. The corresponding two sets of emission lines are in the blue and in the green, respectively, the strongest one being the ${ }^{5} \mathrm{D}_{4}-{ }^{7} \mathrm{~F}_{5}$ transition at $541 \mathrm{~nm}$. This is shown in Fig. 8, where EL spectra for MOS devices implanted with two different doses of $\mathrm{Tb}$ are plotted (the splitting is probably due to the crystal field). The green luminescence increases strongly with $\mathrm{Tb}$ concentration, whereas the blue does not. This can be ascribed to a cross-relaxation mechanism, effectively depopulating the ${ }^{5} \mathrm{D}_{3}$ in favor of the ${ }^{5} \mathrm{D}_{4}$ state [14]. As a result, the most-intense luminescing devices are essentially green emitters.
The rare-earth ions in these MOS structures are impactexcited by hot electrons, which are accelerated in the $\mathrm{SiO}_{2}$ conduction band by the high electric field after FowlerNordheim tunneling through the oxide barrier [40].

We have achieved very high external quantum efficiencies of over $1 \%$ for $\mathrm{SiO}_{2}$ :Gd in the deep UV and $15 \%$ for $\mathrm{SiO}_{2}: \mathrm{Tb}$. Since the latter emits in the visible green spectral region, one can express this also by a luminous efficiency of $2 \mathrm{~lm} / \mathrm{W}$. These values come close to being competitive to nitride based LEDs. Still, the wall plug efficiency is not optimal yet, since due to our relatively thick oxide of $100 \mathrm{~nm}$, the necessary electric fields of $10 \mathrm{MV} / \mathrm{cm}$ are only reached at voltages near $100 \mathrm{~V}$.

\section{Summary}

Efficient MOS type Si based light emitters doped with different rare-earth elements are available today, from the $1.54 \mu \mathrm{m}$ telecom infrared range through the visible up to the deep UV $(316 \mathrm{~nm})$. This paves the way to many applications in fields as various as signal transmission, display technology and bio-sensing, respectively. Si pn junction LEDs enhanced with an integrated microcavity could also become a viable solution for optical interconnects. Si based light emitters may find their niches when efficiency and power are not the most crucial issues, but rather low cost and monolithic integration.

\section{Acknowledgements}

We gratefully acknowledge a collaboration with $\mathrm{S}$. Mantl, U. Breuer (both at Forschungszentrum Jülich, Germany) and S. Winnerl on the resonant cavity diodes, with L. Rebohle and T. Gebel (both at nanoparc GmbH, Dresden) on the MOS devices, and we thank A. Mücklich for TEM analysis.

\section{References}

[1] A. Liu, et al., Nature 427 (2004) 615.

[2] L. Pavesi, J. Phys. Condens. Matter 15 (2003) R1169.

[3] H. Rong, R. Jones, A. Liu, O. Cohen, D. Hak, A. Fang, M. Paniccia, Nature 433 (2005) 725.

[4] L.T. Canham, Appl. Phys. Lett. 57 (1990) 1056.

[5] L. Pavesi, C. Mazzoleni, A. Tredicucci, V. Pellegrini, Appl. Phys. Lett. 67 (1995) 3280.

[6] S. Chan, P.M. Fauchet, Appl. Phys. Lett. 75 (1999) 274.

[7] K.D. Hirschman, L. Tsybeskov, S.P. Duttagupta, P.M. Fauchet, Nature 384 (1996) 338.

[8] J. Engvall, J. Olajos, H.G. Grimmeis, H. Kibbel, H. Presting, Phys. Rev. B 51 (1995) 2001.

[9] G. Franzò, et al., Appl. Phys. A 74 (2002) 1.

[10] L. Pavesi, L. Dal Negro, C. Mazzoleni, G. Franzò, F. Priolo, Nature 408 (2000) 440

[11] A. Polman, J. Appl. Phys. 82 (1997) 1. 
[12] S. Coffa, G. Franzò, F. Priolo, Appl. Phys. Lett. 69 (1996) 2077.

[13] J.M. Sun, W. Skorupa, T. Dekorsy, M. Helm, L. Rebohle, T. Gebel, Appl. Phys. Lett. 85 (2004) 3387.

[14] J.M. Sun, W. Skorupa, T. Dekorsy, M. Helm, L. Rebohle, T. Gebel, J. Appl. Phys. 97 (2005), in print.

[15] M. Fujii, M. Yoshida, Y. Kanzawa, S. Hayashi, K. Yamamoto, Appl. Phys. Lett. 71 (1997) 1198.

[16] F. Iacona, et al., Appl. Phys. Lett. 81 (2002) 3242.

[17] M.E. Castagna, et al., Mater. Sci. Eng. B 105 (2003) 83.

[18] J.M. Sun, W. Skorupa, T. Dekorsy, M. Helm, A.N. Nazarov, Opt. Mater. 27 (2005) 1050.

[19] M.A. Green, J. Zhao, A. Wang, P.J. Reece, M. Gal, Nature 412 (2001) 805.

[20] N.g. Wal Lek, M.A. Lourenço, R.M. Gwilliam, S. Ledain, G. Shao, K.P. Homewood, Nature 410 (2001) 192.

[21] J.M. Sun, T. Dekorsy, W. Skorupa, B. Schmidt, M. Helm, Appl. Phys. Lett. 83 (2003) 3885.

[22] J. Potfajova, J.M. Sun, S. Winnerl, T. Dekorsy, W. Skorupa, B. Schmidt, M. Helm, S. Mantl, U. Breuer, Electron. Lett. 40 (2004) 904.

[23] J.M. Sun, T. Dekorsy, W. Skorupa, B. Schmidt, A. Mücklich, M. Helm, Phys. Rev. B 70 (2004) 155316.

[24] J.M. Sun, T. Dekorsy, W. Skorupa, B. Schmidt, M. Helm, Appl. Phys. Lett. 82 (2003) 2823.
[25] I.A. Buyanova, W.M. Chen, A. Henry, W.X. Ni, G.V. Hansson, B. Monemar, Phys. Rev. B 53 (1996) 9587.

[26] H. Weman, B. Monemar, G.S. Oehrlein, S.J. Jeng, Phys. Rev. B 42 (1990) 3109 .

[27] F. Iacona, G. Franzò, E.C. Moreira, F. Priolo, J. Appl. Phys. 89 (2001) 8354.

[28] D. Sotta, E. Hadji, N. Magnea, E. Delamadeleine, P. Besson, P. Renard, H. Moriceau, J. Appl. Phys. 92 (2002) 2207.

[29] S. Fan, P.R. Villeneuve, J.D. Joannopoulos, E.F. Schubert, Phys. Rev. Lett. 78 (1997) 3294.

[30] M. Zelsmann, et al., Appl. Phys. Lett. 83 (2003) 2542.

[31] M.J. Chen, J.L. Chen, J.Y. Li, J.F. Chang, S.C. Tsai, C.S. Tsai, Appl. Phys. Lett. 84 (2004) 2163.

[32] Y.Q. Wang, A.J. Steckl, Appl. Phys. Lett. 82 (2003) 502.

[33] D.C. Krupka, J. Appl. Phys. 43 (1972) 476.

[34] T. Senda, Y.-J. Cho, T. Hirakawa, H. Okamoto, H. Takakura, Y. Hamakawa, Jpn J. Appl. Phys. 39 (2000) 4716.

[35] A. Chitnis, et al., Appl. Phys. Lett. 82 (2003) 2565; V. Adivaran, et al., Appl. Phys. Lett. 85 (2004) 2175.

[36] A.J. Fischer, et al., Appl. Phys. Lett. 84 (2004) 3394.

[37] A. Yasan, et al., Appl. Phys. Lett. 83 (2003) 4701.

[38] S. Nagahama, et al., Phys. Status Solidi (a) 188 (2001) 1.

[39] Th. Gessmann, E.F. Schubert, J. Appl. Phys. 95 (2004) 2203.

[40] D.J. DiMaria, E. Cartier, D. Arnold, J. Appl. Phys. 73 (1993) 3367. 